\title{
Numerical Experiments Using MATLAB: Superconvergence of Nonconforming Finite Element Approximation for Second-Order Elliptic Problems
}

\author{
Anna Harris ${ }^{1}$, Stephen Harris ${ }^{2}$, Danielle Rauls ${ }^{1}$ \\ ${ }^{1}$ Department of Mathematics and Computer Science, University of Arkansas at Pine Bluff, Pine Bluff, Arkansas, USA \\ ${ }^{2}$ US Food and Drug Administration, National Center for Toxicology Research, Jefferson, Arkansas, USA \\ Email: ^harrisa@uapb.edu, stephen.harris@fda.hhs.gov
}

How to cite this paper: Harris, A., Harris, S. and Rauls, D. (2016) Numerical Experiments Using MATLAB: Superconvergence of Nonconforming Finite Element Approximation for Second-Order Elliptic Problems. Applied Mathematics, 7, 2174-2182. http://dx.doi.org/10.4236/am.2016.717173

Received: September 20, 2016 Accepted: November 19, 2016

Published: November 22, 2016

Copyright $\odot 2016$ by authors and Scientific Research Publishing Inc. This work is licensed under the Creative Commons Attribution International License (CC BY 4.0).

http://creativecommons.org/licenses/by/4.0/ (c) () Open Access

\begin{abstract}
The superconvergence in the finite element method is a phenomenon in which the finite element approximation converges to the exact solution at a rate higher than the optimal order error estimate. Wang proposed and analyzed superconvergence of the conforming finite element method by $L^{2}$-projections. However, since the conforming finite element method (CFEM) requires a strong continuity, it is not easy to construct such finite elements for the complex partial differential equations. Thus, the nonconforming finite element method (NCFEM) is more appealing computationally due to better stability and flexibility properties compared to CFEM. The objective of this paper is to establish a general superconvergence result for the nonconforming finite element approximations for second-order elliptic problems by $L^{2}$-projection methods by applying the idea presented in Wang. MATLAB codes are published at https://github.com/annaleeharris/Superconvergence-NCFEM for anyone to use and to study. The results of numerical experiments show great promise for the robustness, reliability, flexibility and accuracy of superconvergence in NCFEM by $L^{2}$ projections.
\end{abstract}

\section{Keywords}

Nonconforming Finite Element Methods, Superconvergence, $L^{2}$-Projection, Second-Order Elliptic Equation

\section{Introduction}

The conforming finite element method (CFEM) requires a strong continuity; hence it is not easy to construct such finite elements for the complex partial differential equations. 
The nonconforming finite element method (NCFEM) is more appealing computationally due to better stability and flexibility properties compared to CFEM [1] [2] [3]. The superconvergence in the finite element method is a phenomenon in which the finite element approximation converges to the exact solution at a rate higher than the optimal order error estimate. Wang proposed and analyzed superconvergence of the conforming finite element method by $L^{2}$-projections. The main idea behind the $L^{2}$-projections is to project the finite element solution to another finite element space with a coarse mesh and a higher order of polynomials.

The objective of this paper is to establish a general superconvergence result for the nonconforming finite element approximations for second-order elliptic problems by $L^{2}$-projection methods by applying the idea presented in Wang [4].

This paper is organized as follows. In Section 2, we present a review for the nonconforming finite element method for the second-order elliptic problem. In Section 3, we develop a general theory of superconvergence by following the idea presented in Wang [4]. In Section 4, we perform numerical experiments to support the theoretical results. Numerical experiements of superconvergence of NCFEM are performed in MATLAB and its codes are posted at https://github.com/annaleeharris/Superconvergence-NCFEM for anyone to use and to study.

\section{NCFEM for the Second-Order Elliptic Problem}

Consider the second-order elliptic problem with the Dirichlet boundary condition which seeks $u \in H^{1}(\Omega)$ satisfying

$$
\begin{aligned}
& \Delta u=f \quad \text { in } \Omega, \\
& u=0 \quad \text { on } \partial \Omega,
\end{aligned}
$$

where $\Delta$ is the Laplacian operator, $\Omega$ is a bounded, connected, and open subset of $R^{2}, \partial \Omega$ is a Lipschitz continuous boundary, and a given function $f$ is the external force.

A variational formulation of (1) seeks $u \in H_{0}^{1}(\Omega)$ such that

$$
a(u, v)=(f, v), \quad \forall v \in H_{0}^{1}(\Omega),
$$

where

$$
a(u, v)=(\nabla u, \nabla v)=\int_{\Omega} \nabla u \cdot \nabla v \mathrm{~d} \Omega
$$

Let $\mathcal{T}_{h}$ be a quasi-uniform, i.e., it is regular and satisfies the inverse assumption [5], triangulation of $\Omega$ with $\operatorname{diam}(K) \leq h, K \in \mathcal{T}_{h}$. Let $P_{k}(K)$ be the space of polynomials of degree at most $k$ with $k \geq 0$ on $K$. Let $\varepsilon_{h}$ denote the union of the boundaries of all elements $K \in \mathcal{T}_{h}$ and let $\varepsilon_{h}^{0}=\varepsilon_{h} \backslash \partial \Omega$ be the collection of all interior edges. Assume that the polynomial space in the construction of $V_{h}$ contains $P_{k}(K)$, $k \geq 1$. Define the finite element space $V_{h}$ associated with $\mathcal{T}_{h}$ as

$$
\begin{aligned}
V_{h}=\left\{v \in L^{2}(\Omega):\left.v\right|_{K} \in P_{k}(K), \forall K \in \mathcal{T}_{h}, v\right. \text { is continuous at the middle point of } \\
\\
\left.e \in \varepsilon_{h}^{0}, \text { and } v \text { is zero at the middle point of boundary edge } e \text { on } \partial \Omega\right\} .
\end{aligned}
$$


The finite element space $V_{h}$ is assumed to satisfy the following approximation property for any $u \in H^{m+1}(\Omega)[6]$ :

$$
\inf _{v \in V_{h}}\left(\|u-v\|+h\|u-v\|_{1}\right) \leq C h^{m+1}\|u\|_{m+1}, \quad 0 \leq m \leq k .
$$

The nonconforming finite element approximation problem (2) seeks $u_{h} \in V_{h}$ such that

$$
a_{h}\left(u_{h}, v\right)=(f, v), \quad \forall v \in V_{h},
$$

where

$$
a_{h}\left(u_{h}, v\right)=\sum_{K \in \mathcal{T}_{h}}\left(\nabla u_{h}, \nabla v\right)_{K}=\sum_{K \in \mathcal{T}_{h}} \int_{K} \nabla u_{h} \cdot \nabla v \mathrm{~d} x
$$

A well known error estimate for the finite element approximation solution $u_{h}$ is the following [7]:

$$
\left\|u-u_{h}\right\|+h\left\|u-u_{h}\right\|_{1, h} \leq C h^{k+1}\|u\|_{k+1},
$$

where $\mathrm{C}$ is a constant independent of the mesh size $h$.

To apply the superconvergence of finite element approximation, we assume that domain $\Omega$ is so regular that it ensures a $H^{s}, s \geq 1$, regularity for the solution of (2). In other words, for any $f \in H^{s-2}(\Omega)$ the problem (2) has a unique solution $u \in H_{0}^{1}(\Omega)$ satisfying the following a priori estimate

$$
\|u\|_{s} \leq C\|f\|_{s-2}, \quad \forall f \in H^{s-2}(\Omega), s \geq 1,
$$

where $C$ is a constant independent of data $f$.

\section{Superconvergence of NCFEM}

Let $\mathcal{T}_{\tau}$ be another finite element partition with coarse mesh size $\tau$ where $h \ll \tau$. Assume that $\tau$ and $h$ have the following relation:

$$
\tau=h^{\alpha}, \quad \alpha \in(0,1) .
$$

Let $V_{\tau}$ be any finite element space consisting of piecewise polynomial of degree $r$ associated with the partition $\mathcal{T}_{\tau}$. Define $Q_{\tau}$ to be the $L^{2}$-projection from $L^{2}(\Omega)$ onto the finite element space $V_{\tau}$. The finite element space $V_{\tau}$ is defined as follows:

$$
V_{\tau}=\left\{v \in L^{2}(\Omega):\left.v\right|_{K} \in P_{r}(K), \forall K \in \mathcal{T}_{\tau}\right\} .
$$

The following lemma will provide an error estimate for $Q_{\tau} u-Q_{\tau} u_{h}$.

Lemma 1 Assume that the second-order elliptic problem (2) holds (5) with $1 \leq s \leq k+1$ and $V_{\tau} \subset H^{s-2}(\Omega)$. Then there exists a constant $C$ independent of $h$ and $\tau$ such that

$$
\left\|Q_{\tau} u-Q_{\tau} u_{h}\right\| \leq C h^{k+s-1+\alpha \min (0,2-s)}\|u\|_{k+1},
$$

where $\alpha \in(0,1)$ and $\tau \gg h$. 
Proof. Using the definition of $\|\cdot\|$ and $Q_{\tau}$, we have

$$
\left\|Q_{\tau} u-Q_{\tau} u_{h}\right\|=\sup _{\phi \in L^{2}(\Omega),\|\phi\|=1}\left|\left(Q_{\tau} u-Q_{\tau} u_{h}, \phi\right)\right|
$$

and

$$
\left(Q_{\tau} u-Q_{\tau} u_{h}, \phi\right)=\left(u-u_{h}, Q_{\tau} \phi\right)
$$

Then

$$
\left\|Q_{\tau} u-Q_{\tau} u_{h}\right\|=\sup _{\phi \in L^{2}(\Omega),\|\phi\|=1}\left|\left(u-u_{h}, Q_{\tau} \phi\right)\right| .
$$

Consider the following problem:

$$
\begin{aligned}
& -\Delta w=Q_{\tau} \phi \quad \text { in } \Omega, \\
& w=0 \quad \text { on } \partial \Omega .
\end{aligned}
$$

Multiplying the second-order elliptic Equation (1) by $v$ and integrating it over $\Omega$ give

$$
a_{h}(u, v)-\sum_{K \in \mathcal{I}_{h}}\langle\nabla u \cdot \boldsymbol{n}, v\rangle_{\partial K}=(f, v),
$$

where $n$ is the unit outward normal.

Subtract (3) from the above Equation (10) gives

$$
a_{h}\left(u-u_{h}, v\right)=\sum_{K \in T_{h}}\langle\nabla u \cdot \boldsymbol{n}, v\rangle_{\partial K}, \quad \forall v \in V_{h} .
$$

Multiplying (9) by $u-u_{h}$, integrating it over $\Omega$, adding and subtracting $w_{I} \in V_{h}$, and using the result (11) we have

$$
\begin{aligned}
\left(Q_{\tau} \phi, u-u_{h}\right) & =\left(-\Delta w, u-u_{h}\right) \\
& =a_{h}\left(w, u-u_{h}\right)-\sum_{K \in \mathcal{T}_{h}}\left\langle\nabla w \cdot \boldsymbol{n}, u-u_{h}\right\rangle_{\partial K} \\
& =a_{h}\left(w-w_{I}+w_{I}, u-u_{h}\right)-\sum_{K \in \mathcal{T}_{h}}\left\langle\nabla w \cdot \boldsymbol{n}, u-u_{h}\right\rangle_{\partial K} \\
& =a_{h}\left(w-w_{I}, u-u_{h}\right)+a_{h}\left(w_{I}, u-u_{h}\right)-\sum_{K \in \mathcal{T}_{h}}\left\langle\nabla w \cdot \boldsymbol{n}, u-u_{h}\right\rangle_{\partial K} \\
& =a_{h}\left(w-w_{I}, u-u_{h}\right)+\sum_{K \in \mathcal{T}_{h}}\left\langle\nabla u \cdot \boldsymbol{n}, w_{I}\right\rangle_{\partial K}-\sum_{K \in \mathcal{T}_{h}}\left\langle\nabla w \cdot \boldsymbol{n}, u-u_{h}\right\rangle_{\partial K} .
\end{aligned}
$$

The line integrals of the above equations are approximated in [6] as follows:

$$
\begin{aligned}
& \sum_{K \in \mathcal{T}_{h}}\left\langle\nabla u \cdot \boldsymbol{n}, w_{I}\right\rangle_{\partial K} \leq C h^{k+s-1}\|u\|_{k+1}\|w\|_{s}, \\
& \sum_{K \in \mathcal{T}_{h}}\left\langle\nabla w \cdot \boldsymbol{n}, u-u_{h}\right\rangle_{\partial K} \leq C h^{k+s-1}\|u\|_{k+1}\|w\|_{s} .
\end{aligned}
$$

Using the Cauchy-Schwartz inequality, the approximation property (2), and line integral approximations (12) and (13) we have

$$
\begin{aligned}
\left|\left(Q_{\tau} \phi, u-u_{h}\right)\right| & =a_{h}\left(w-w_{I}, u-u_{h}\right)+\sum_{K \in \mathcal{T}_{h}}\left\langle\nabla u \cdot \boldsymbol{n}, w_{I}\right\rangle_{\partial K}-\sum_{K \in \mathcal{T}_{h}}\left\langle\nabla w \cdot \boldsymbol{n}, u-u_{h}\right\rangle_{\partial K} \\
& \leq\left\|w-w_{I}\right\|\left\|u-u_{h}\right\|+C h^{k+s-1}\|u\|_{k+1}\|w\|_{s} \\
& \leq C h^{k+s-1}\|w\|_{s}\|u\|_{k+1} .
\end{aligned}
$$


Substituting $\|w\|_{s}$ as $\left\|Q_{\tau} \phi\right\|_{s-2}$ by the $H^{s}$ regularity, applying the inverse inequality to the term $\left\|Q_{\tau} \phi\right\|_{s-2}$ and using the definition of $\tau=h^{\alpha}$ we have

$$
\begin{aligned}
\left|\left(Q_{\tau} \phi, u-u_{h}\right)\right| & \leq C h^{k+s-1}\left\|Q_{\tau} \phi\right\|_{s-2}\|u\|_{k+1} \\
& \leq C h^{k+s-1} \tau^{\min (0,2-s)}\left\|Q_{\tau} \phi\right\|\|u\|_{k+1} \\
& \leq C h^{k+s-1} \tau^{\min (0,2-s)}\|\phi\|\|u\|_{k+1} \\
& \leq C h^{k+s-1+\alpha \min (0,2-s)}\|u\|_{k+1} .
\end{aligned}
$$

Combining the above equation with the Equation (8) we have

$$
\left\|Q_{\tau} u-Q_{\tau} u_{h}\right\| \leq C h^{k+s-1+\alpha \min (0,2-s)}\|u\|_{k+1},
$$

which completes the proof of the lemma.

The following theorem provides an error estimate for $u-Q_{\tau} u_{h}$.

Theorem 1 Assume that (5) holds true with $1 \leq s \leq k+1$ and $V_{\tau} \subset H^{s-2}(\Omega)$. If $u_{h}$ is the finite element approximation of the exact solution $u \in H^{k+1}(\Omega) \cap H^{r+1}(\Omega)$ $\cap H_{0}^{1}(\Omega)$ of (2), then there exists a constant $C$ independent of $h$ and $\tau$ such that

$$
\begin{aligned}
& \left\|u-Q_{\tau} u_{h}\right\|+h^{\alpha}\left\|\nabla_{\tau}\left(u-Q_{\tau} u_{h}\right)\right\| \\
& \leq C h^{\alpha(r+1)}\|u\|_{r+1}+C h^{k+s-1+\alpha \min (0,2-s)}\|u\|_{k+1} .
\end{aligned}
$$

Proof. Since we assume the exact solution $u$ is sufficiently smooth and by the definitions of $Q_{\tau}$ and $\tau$, we have

$$
\left\|u-Q_{\tau} u_{h}\right\| \leq C \tau^{r+1}\|u\|_{r+1}=C h^{\alpha(r+1)}\|u\|_{r+1} .
$$

Using the triangle inequality and combining (16) and Lemma 1 we obtain

$$
\begin{aligned}
\left\|u-Q_{\tau} u_{h}\right\| & \leq\left\|u-Q_{\tau} u\right\|+\left\|Q_{\tau} u-Q_{\tau} u_{h}\right\| \\
& \leq C h^{\alpha(r+1)}\|u\|_{r+1}+C h^{k+s-1+\alpha \min (0,2-s)}\|u\|_{k+1},
\end{aligned}
$$

which completes the error estimate of $\left\|u-Q_{\tau} u_{h}\right\|$.

Similarly, we estimate $h^{\alpha}\left\|\nabla_{\tau}\left(u-Q_{\tau} u_{h}\right)\right\|$.

Using the inverse inequality and the definitions of $Q_{\tau}$ and $\tau$ we have

$$
\left\|\nabla_{\tau}\left(u-Q_{\tau} u_{h}\right)\right\| \leq C \tau^{r}\|u\|_{r+1}=C h^{\alpha r}\|u\|_{r+1} .
$$

Using the triangle inequality and combining (17) and Lemma 1 we have

$$
\begin{aligned}
& h^{\alpha}\left\|\nabla_{\tau}\left(u-Q_{\tau} u_{h}\right)\right\| \leq h^{\alpha}\left\|\nabla_{\tau} u-\nabla_{\tau} Q_{\tau} u\right\|+h^{\alpha}\left\|\nabla_{\tau} Q_{\tau} u-\nabla_{\tau} Q_{\tau} u_{h}\right\| \\
& \leq C h^{\alpha(r+1)}\|u\|_{r+1}+C h^{k+s-1+\alpha \min (0,2-s)}\|u\|_{k+1} .
\end{aligned}
$$

Hence the theorem has been proved.

The optimal $\alpha$ is selected using Theorem 1 for the error estimates:

$$
\begin{aligned}
& \alpha(r+1)=k+s-1+\alpha \min (0,2-s), \\
& \alpha=\frac{k+s-1}{r+1-\min (0,2-s)} .
\end{aligned}
$$




\section{Numerical Experiments of Superconvergence of NCFEM by $L^{2}$-Projection Methods}

In this section, we present numerical experiments for second-order elliptic problems to support our theoretical results. Assume that the exact solution of the second-order elliptic problem has the $H^{s}$ regularity for some $1 \leq s \leq 2$ and for simplicity, assume $k=1, s=2$, and $r=2$ which gives $\alpha=\frac{2}{3}$ using the $\alpha$ formula (18).

From the theoretical result (15) we have the following optimal error estimates:

$$
\left\|u-Q_{\tau} u_{h}\right\| \leq C h^{\alpha(r+1)}\|u\|_{r+1}+C h^{k+s-1+\alpha \min (0,2-s)}\|u\|_{k+1} \leq C h^{2}\|u\|_{3}
$$

and

$$
\left\|\nabla_{\tau}\left(u-Q_{\tau} u_{h}\right)\right\| \leq C h^{\alpha r}\|u\|_{r+1}+C h^{k+s-1-\alpha+\alpha \min (0,2-s)-\alpha}\|u\|_{k+1} \leq C h^{\frac{4}{3}}\|u\|_{3} .
$$

From the results (19) and (20), theoretically, in $L^{2}$ norm the $L^{2}$-projection to the existing numerical approximation does not improve the convergence rate but in $H_{1}$ norm the $L^{2}$-projection to the existing numerical solution provides some superconvergence.

The finite element partition $\mathcal{T}_{h}$ is constructed by dividing the domain into an $n^{3} \times n^{3}$ rectangular mesh then dividing the rectangular mesh with the positive slope to form two triangles. The coarse finite element partition $\mathcal{T}_{\tau}$ is also constructed by dividing the domain into an $n^{2} \times n^{2}$ rectangular mesh then dividing the rectangular mesh with the positive slope to form two triangles. The finite element space $V_{h}$ consists of the space of the linear polynomials $P_{1}(K)$ associated with the partition $\mathcal{T}_{h}$ and the dual finite element space $V_{\tau}$ consists of the space of the quadratic polynomials $P_{2}(K)$ associated with the partition $\mathcal{T}_{\tau}$. The finite element spaces $V_{h}$ and $V_{\tau}$ are defined as follows:

$$
\begin{gathered}
V_{h}=\left\{v \in L^{2}(\Omega):\left.v\right|_{K} \in P_{k}(K), \forall K \in \mathcal{T}_{h}, v\right. \text { is continuous at the middle point of } \\
\left.e \in \varepsilon_{h}^{0}, \text { and } v \text { is zero at the middle point of boundary edge } e \text { on } \partial \Omega\right\} .
\end{gathered}
$$

and

$$
V_{\tau}=\left\{v \in L^{2}(\Omega):\left.v\right|_{K} \in P_{2}(K), \forall K \in \mathcal{T}_{\tau}\right\}
$$

The numerical approximation is refined as $h=n^{-3}$ where $n=2, \cdots, 6$. The length of $\tau=n \cdot h, n=2, \cdots, 6$ and each $\tau$ element contains $n^{2} \cdot h$ elements.

Using the $\alpha$ Equation (18) and our choice of $k=1, s=2$, and $r=2$ we have

$$
\alpha=\frac{k+s-2}{r+1-\min (0,2-s)}=\frac{2}{3} \text {. }
$$

Using the difference in mesh size and a higher degree of polynomials we shall produce some superconvergence of NCFEM for the second-order elliptic problems.

Example 1 . Let the domain $\Omega=[0,1] \times[0,1]$ and the exact solution is assumed to be as follows: 


$$
u=x(1-x) y(1-y) .
$$

From Table 1 we observe that the $L^{2}$-projection to the existing numerical approximation $u_{h}$ reduced the error estimates in $L^{2}$ norm and in $H_{1}$ norm. In $L^{2}$ norm the convergence rate of $\left\|u-Q_{\tau} u_{h}\right\|$ is similar to the convergence rate of $\left\|u-u_{h}\right\|$ which is the same as the theoretical result (19). The convergence rate of $\left|u-Q_{\tau} u_{h}\right|_{1}$ is about $33 \%$ faster than the convergence rate of $\left|u-u_{h}\right|_{1}$ in $H_{1}$ norm (see Figure 2). The surface plots of $Q_{\tau} u_{h}$ in coarse meshes and $u_{h}$ in fine meshes are shown in Figure 1. The numerical example 1 clearly supports the theoretical result and confirms the superconvergence of NCFEM for the second-order elliptic problem.

Example 2. Let the domain $\Omega=[0,1] \times[0,1]$ and let the analytical solution be given as

$$
u=x(1-x) y \cos (1.5 \pi y)
$$

From Table 2, we can see that the numerical example 2 supports the theoretical result (15). See Figure 3, when $h=3^{-3}$ and $\tau=3^{-2}$, we can project $3^{2}$ fine triangle elements onto one coarse triangle element. Thus, as $n$ increases, we can project $n^{2}$ more fine triangle elements to one coarse triangle element in which the process of refining elements produces better error estimates. The $L^{2}$-projection to the existing numerical approximation $u_{h}$ produced some superconvergence in $H_{1}$ norm and did not affect the convergence rate in $L^{2}$ norm (see Figure 4). The numerical example 2 also

Table 1. Numerical error approximation results using NCFEM in Example 1, $u=x(1-x) y(1-y)$.

\begin{tabular}{cccccc}
\hline iter & $h$ & $\left\|\nabla_{h}\left(u-u_{h}\right)\right\|$ & $\left\|u-u_{h}\right\|$ & $\left\|\nabla_{\tau}\left(u-Q_{\tau} u_{h}\right)\right\|$ & $\left\|u-Q_{\tau} u_{h}\right\|$ \\
\hline 1 & $2^{-3}$ & $0.1388 \mathrm{e}-1$ & $0.3909 \mathrm{e}-3$ & $0.8184 \mathrm{e}-2$ & $0.3920 \mathrm{e}-3$ \\
2 & $3^{-3}$ & $0.4138 \mathrm{e}-2$ & $0.3443 \mathrm{e}-4$ & $0.1635 \mathrm{e}-2$ & $0.3431 \mathrm{e}-4$ \\
3 & $4^{-3}$ & $0.1747 \mathrm{e}-2$ & $0.6104 \mathrm{e}-5$ & $0.5190 \mathrm{e}-3$ & $0.6104 \mathrm{e}-5$ \\
4 & $5^{-3}$ & $0.8944 \mathrm{e}-3$ & $0.1600 \mathrm{e}-5$ & $0.2127 \mathrm{e}-3$ & $0.1600 \mathrm{e}-5$ \\
5 & $6^{-3}$ & $0.5176 \mathrm{e}-3$ & $0.5358 \mathrm{e}-6$ & $0.1026 \mathrm{e}-3$ & $0.5359 \mathrm{e}-6$ \\
& $O\left(h^{r}\right)$ & 0.9981 & 2.000 & 1.3287 & 2.0010 \\
\hline
\end{tabular}
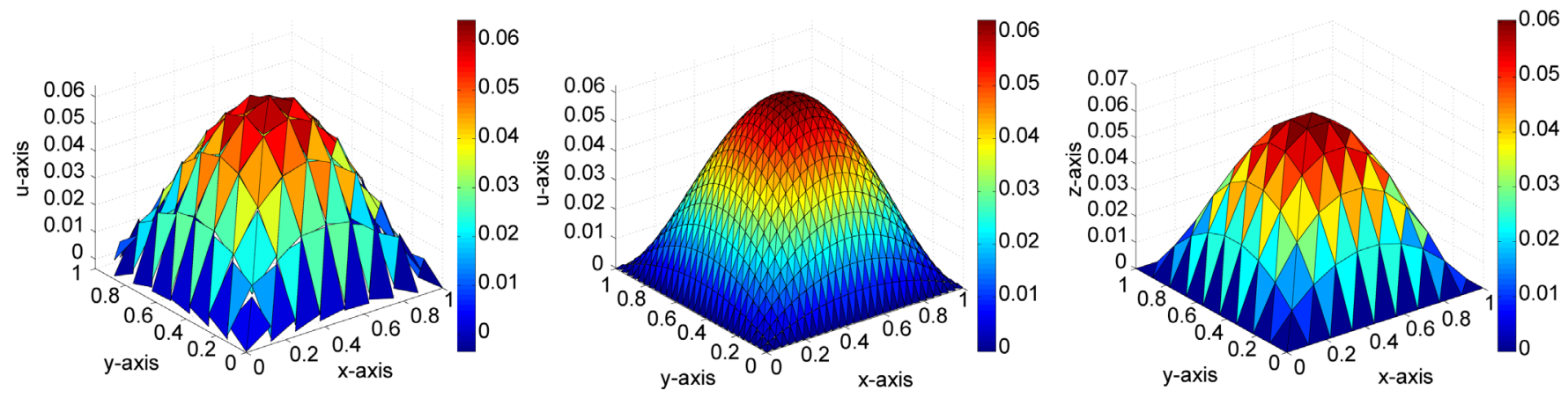

Figure 1. Surface plots of approximation using NCFEM in Example 1, $u=x(1-x) y(1-y)$. (L): Surface plot of $u_{h}$ when $h=2^{-3}$. (M): Surface of plot of $u_{h}$ when $h=3^{-3}$. (R): Surface plot of $Q_{\tau} u_{h}$ when $\tau=3^{-2}$. 

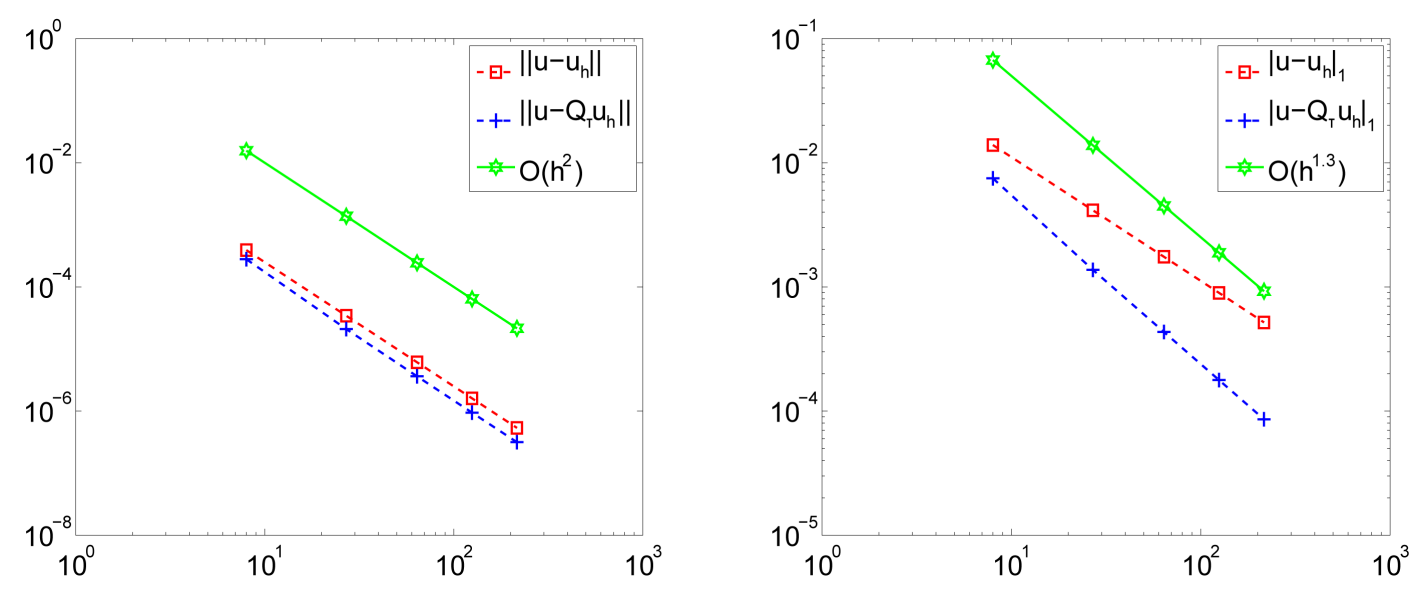

Figure 2. Error convergence rates using NCFEM in Example 1, $u=x(1-x) y(1-y)$. (L): $L^{2}$ norm error; (R): $H_{1}$ norm error.

Table 2. Numerical error approximation results using NCFEM in Example 2, $u=x(1-x) y \cos (1.5 \pi y)$.

\begin{tabular}{cccccc}
\hline iter & $h$ & $\left\|\nabla_{h}\left(u-u_{h}\right)\right\|$ & $\left\|u-u_{h}\right\|$ & $\left\|\nabla_{\tau}\left(u-Q_{\tau} u_{h}\right)\right\|$ & $0.2214 \mathrm{e}-1$ \\
\hline 1 & $2^{-3}$ & $0.3933 \mathrm{e}-1$ & $0.8429 \mathrm{e}-3$ & $0.8453 \mathrm{e}-3$ \\
2 & $3^{-3}$ & $0.1189 \mathrm{e}-1$ & $0.7404 \mathrm{e}-4$ & $0.7408 \mathrm{e}-4$ \\
3 & $4^{-3}$ & $0.5019 \mathrm{e}-2$ & $0.1317 \mathrm{e}-4$ & $0.1392 \mathrm{e}-2$ & $0.1318 \mathrm{e}-4$ \\
4 & $5^{-3}$ & $0.2570 \mathrm{e}-2$ & $0.3454 \mathrm{e}-5$ & $0.5708 \mathrm{e}-3$ & $0.3455 \mathrm{e}-5$ \\
5 & $6^{-3}$ & $0.1487 \mathrm{e}-2$ & $0.1156 \mathrm{e}-5$ & $1.2754 \mathrm{e}-3$ & $0.1157 \mathrm{e}-5$ \\
& $O\left(h^{r}\right)$ & 0.9983 & 1.9998 & 2.0006 \\
\hline
\end{tabular}
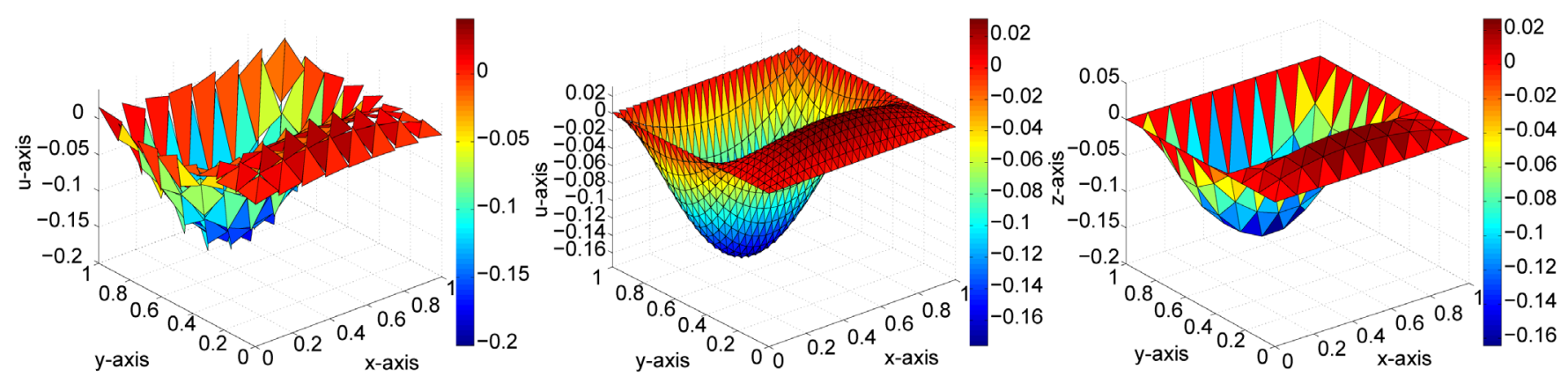

Figure 3. Surface plots of approximation using NCFEM in Example 2, $u=x(1-x) y \cos (1.5 \pi y)$. (L): Surface plot of $u_{h}$ when $h=2^{-3}$. (M): Surface of plot of $u_{h}$ when $h=3^{-3}$. (R): Surface plot of $Q_{\tau} u_{h}$ when $\tau=3^{-2}$.

supports the theoretical result and confirms the superconvergence of NCFEM for the second-order elliptic problem.

\section{Conclusion}

The $L^{2}$-projection to the existing numerical approximation $u_{h}$ produced some superconvergence in $H_{1}$ norm, convergence rate $\geq 1.3$, but did not affect the convergence 

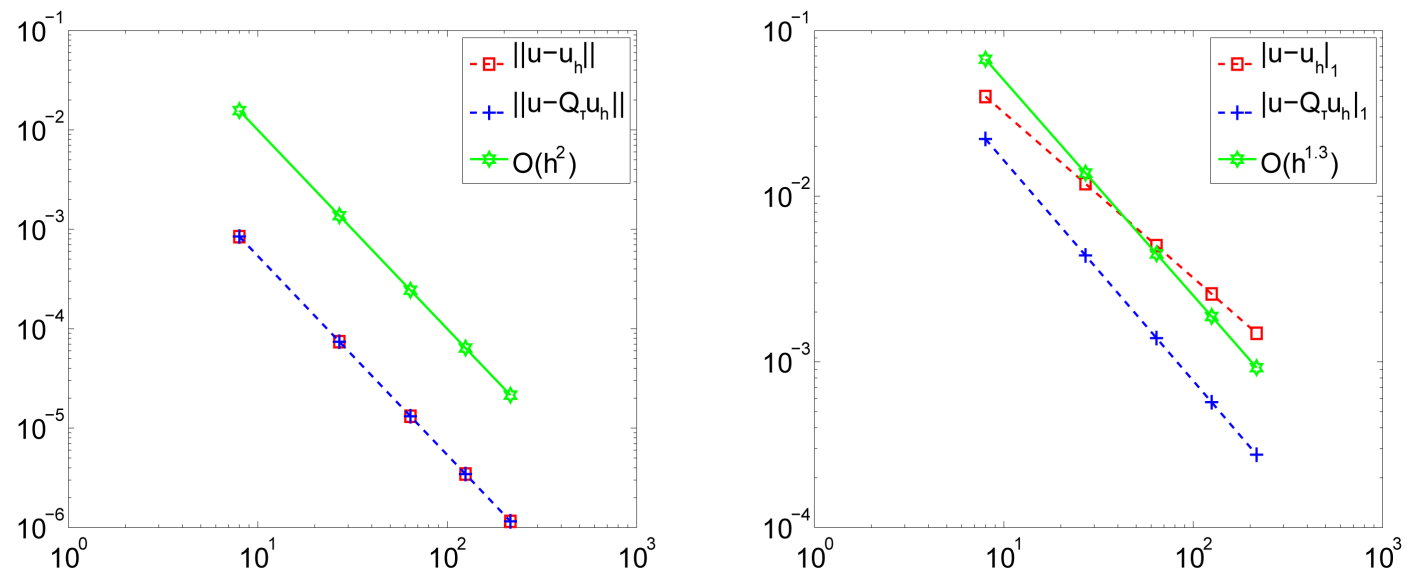

Figure 4. Error convergence rates using NCFEM in Example 2, $u=x(1-x) y \cos (1.5 \pi y)$. (L): $L^{2}$ norm error; (R): $H_{1}$ norm error.

rate in $L^{2}$ norm. With the numerical experiments we can conclusively support the theoretical result and confirm the superconvergence of NCFEM for second-order elliptic problems by $L^{2}$-projection method.

\section{Acknowledgements}

We thank the Editor and the peer-reviewers for their comments. Research of Anna Harris is funded by the National Science Foundation Historical Black Colleges and Universities Undergraduate Program Research Initiative Award grant (\#1505119). This support is greatly appreciated.

\section{References}

[1] Croouzeix, M. and Raviart, P.A. (1973) Conforming and Nonconforming Finite Element Methods for Solving the Stationary Stokes Equations. R.A.I.R.O. R, 3, 33-76.

[2] Douglas Jr, J., Santos, J.E., Sheen, D. and Ye, X. (1999) Nonconforming Galerkin Methods Based on Quadrilateral Elements for Second Order Elliptic Problems. Mathematical Modelling and Numerical Analysis, 33, 747-770. https:/doi.org/10.1051/m2an:1999161

[3] Girault, V. and Raviart, P.A. (1986) Finite Element Methods for the Navier-Stokes Equations: Theory and Algorithms. Springer, Berlin. https:/doi.org/10.1007/978-3-642-61623-5

[4] Wang, J. (2000) A Superconvergence Analysis for Finite Element Solutions by the LeastSquare Surface Fitting on Irregular Meshes for Smooth Problems. Journal of Mathematical Study, 33, 229-243.

[5] Ciarlet, P.G. (1978) The Finite Element Method for Elliptic Problems. North-Holland, New York.

[6] Ye, X. (2002) Superconvergence of Nonconforming Finite Element Method for the Stokes Equation. Numer. Method for PDE, 18, 143-154. https:/doi.org/10.1002/num.1036

[7] Brenner, S.C. and Scott, L.R. (1994) The Mathematical Theory of Finite Element Methods. Springer-Verlag, Berlin. https:/doi.org/10.1007/978-1-4757-4338-8 
Submit or recommend next manuscript to SCIRP and we will provide best service for you:

Accepting pre-submission inquiries through Email, Facebook, LinkedIn, Twitter, etc.

A wide selection of journals (inclusive of 9 subjects, more than 200 journals)

Providing 24-hour high-quality service

User-friendly online submission system

Fair and swift peer-review system

Efficient typesetting and proofreading procedure

Display of the result of downloads and visits, as well as the number of cited articles

Maximum dissemination of your research work

Submit your manuscript at: http://papersubmission.scirp.org/

Or contact am@scirp.org 